\title{
Correction to: A New Proof of the Sharpness of the Phase Transition for Bernoulli Percolation and the Ising Model
}

\author{
Hugo Duminil-Copin, Vincent Tassion \\ Département de Mathématiques, Université de Genève, Geneva, Switzerland. \\ E-mail: hugo.duminil@unige.ch; vincent.tassion@unige.ch
}

Published online: 23 March 2018 - @ Springer-Verlag GmbH Germany, part of Springer Nature 2018

\section{Correction to: Commun. Math. Phys. 343, 725-745 (2016) https://doi.org/10.1007/s00220-015-2480-z}

The differential inequality (2.5) should be replaced by

$$
\frac{d}{d \beta}\left\langle\sigma_{0}\right\rangle_{\lambda, \beta, h}^{2} \geq \frac{2 c(\Lambda)}{\beta} \inf _{S \ni 0} \varphi_{\beta}(S)\left(1-\left\langle\sigma_{0}\right\rangle_{\lambda, \beta, h}^{2}\right)-\epsilon(\Lambda, \beta, h)
$$

where $\epsilon(\Lambda, \beta, h):=2 \sum_{x \in \Lambda} \sum_{y \in V \backslash \Lambda} J_{x, y}\left(\left\langle\sigma_{0} \sigma_{x}\right\rangle_{\lambda, \beta, h}-\left\langle\sigma_{0}\right\rangle_{\lambda, \beta, h}\left\langle\sigma_{x}\right\rangle_{\lambda, \beta, h}\right)$.

The correcting term $\epsilon(\Lambda, \beta, h)$ comes from the fact that the summation on $y \notin S$ in the displayed equations before (2.14) should be understood as a summation on $y \in \Lambda \backslash S$. Thus, in (2.14), the term $\frac{1}{\beta} \varphi_{\beta}(S)$ should be replaced by $\frac{1}{\beta} \varphi_{\beta}(S)-\sum_{x \in \Lambda} \sum_{y \in V \backslash \Lambda} J_{x, y}$ $\left\langle\sigma_{0} \sigma_{x}\right\rangle_{S, \beta, 0}$. One then sums on every possible value of $S$ and uses the switching lemma to obtain $\epsilon(\Lambda, \beta, h)$.

The appearance of this correcting term does not modify the proof since the GHS inequality [GHS70] classically implies that $\epsilon(\Lambda, \beta, h)$ tends to 0 as $\Lambda$ tends to $V$.

Independently, another place of the paper can benefit from a slight modification: the definition of $\chi_{n}(\beta)$ on the bottom of Page 743 should be replaced by

$$
\chi_{n}(\beta):=\sup \left\{\sum_{x \in \Lambda}\left\langle\sigma_{0} \sigma_{x}\right\rangle_{\beta}^{+}: \Lambda \subset V \text { with }|\Lambda| \leq n\right\} .
$$

Acknowledgements. We thank Alexander Glazman and Marcin Lis for pointing out the mistake to us. 


\section{Reference}

[GHS70] Griffiths, R.B., Hurst, C.A., Sherman, S.: Concavity of magnetization of an ising ferromagnet in a positive external field. J. Math. Phys. 11, 790-795 (1970)

Communicated by F. Toninelli 